\title{
HYDRODYNAMIC EFFECT ON THE THREE-DIMENSIONAL FLOW PAST A VERTICAL POROUS PLATE
}

\author{
M. GURIA AND R. N. JANA
}

Received 11 April 2005

The study of unsteady hydrodynamic free convective flow of a viscous incompressible fluid past a vertical porous plate in the presence of a variable suction has been made. Approximate solutions have been derived for the velocity and temperature fields, shear stress, and rate of heat transfer using perturbation technique. It is observed that main fluid velocity decreases with increase in Prandtl number, while it increases with increase in suction parameter. The cross-velocity decreases near the plate and increases away from the plate with increase in suction parameter. On the other hand, it increases near the plate and decreases away from the plate with increase in frequency parameter. The amplitude and the tangent of phase shift of the shear stress due to main flow decrease with increase in either Prandtl number, Grashof number, or frequency parameter. It is seen that the temperature decreases with increase in either suction parameter, Prandtl number, or frequency parameter. It is also seen that the amplitude of the rate of heat transfer increases and the tangent of phase shift of rate of heat transfer decreases with increase in Prandtl number.

\section{Introduction}

The research area of laminar flow is continuously growing, and it is the subject of intensive studies in recent years because of its application in engineering, particularly in aeronautical engineering. One of the most important application of laminar flow is the calculation of friction drag of bodies in a flow, for example, the drag of a plate at zero incidence, the friction drag of ship, an airfoil. It is also important for heat transfer between a body and the fluid around it. The effect of different arrangements and configurations of the suction holes and slits on the drag has been studied by various scholars. Misra et al. [4] have studied the effect of buoyancy forces on the three-dimensional flow and heat transfer along a porous vertical plate. Misra et al. [3] also studied the flow of viscous incompressible fluid along an infinite porous plate by applying the transverse sinusoidal suction velocity distribution fluctuating with time. Later, Singh [2] extended this idea by applying transverse sinusoidal suction velocity in the presence of viscous dissipative heat. 
Singh [1] also discussed the effect of magnetic field on the three-dimensional flow past a porous plate.

The aim of this note is to study the effect of buoyancy forces and time-dependent periodic suction on three-dimensional flow past a vertical porous plate. The velocity field, shear stress, temperature distribution, and the rate of heat transfer have been derived. It is observed that main fluid velocity decreases with increase in Prandtl number while it increases with increase in suction parameter. The cross-velocity decreases near the plate and increases away from the plate with increase in suction parameter. On the other hand, it increases near the plate and decreases away from the plate with increase in frequency parameter. The amplitude and the tangent of phase shift of the shear stress due to main flow decrease with increase in either Prandtl number, Grashof number, or frequency parameter. It is seen that the temperature decreases with increase in either Prandtl number, suction parameter, or frequency parameter. It is also seen that the amplitude of the rate of heat transfer increases and the tangent of phase shift of rate of heat transfer decreases with increase in Prandtl number.

\section{Formulation of the problem}

Consider the unsteady flow of viscous, incompressible fluid past along a semi-infinite vertical porous plate. Here, the $x^{\star}$-axis is chosen along the vertical plate, that is, the direction of the flow, $y^{\star}$-axis is perpendicular to the plate, and $z^{\star}$-axis is normal to the $x^{\star} y^{\star}$-plane.

The plate is subjected to periodic suction velocity distribution of the form

$$
v^{\star}=-V_{0}\left[1+\epsilon \cos \left(\frac{\pi u_{\infty} z^{\star}}{v}-c t^{\star}\right)\right]
$$

where $\epsilon(\ll 1)$ is the amplitude of the suction velocity. Denoting velocity components $u^{\star}, v^{\star}, w^{\star}$ in the directions $x^{\star}-, y^{\star}-, z^{\star}$-axes, respectively, the flow is governed by the following equations:

$$
\begin{gathered}
\frac{\partial v^{\star}}{\partial y^{\star}}+\frac{\partial w^{\star}}{\partial z^{\star}}=0 \\
\frac{\partial u^{\star}}{\partial t^{\star}}+v^{\star} \frac{\partial u^{\star}}{\partial y^{\star}}+w^{\star} \frac{\partial u^{\star}}{\partial z^{\star}}=v\left(\frac{\partial^{2} u^{\star}}{\partial y^{\star 2}}+\frac{\partial^{2} u^{\star}}{\partial z^{\star 2}}\right)+g \beta\left(T-T_{\infty}\right), \\
\frac{\partial v^{\star}}{\partial t^{\star}}+v^{\star} \frac{\partial v^{\star}}{\partial y^{\star}}+w^{\star} \frac{\partial v^{\star}}{\partial z^{\star}}=-\frac{1}{\rho} \frac{\partial p^{\star}}{\partial y^{\star}}+v\left(\frac{\partial^{2} v^{\star}}{\partial y^{\star 2}}+\frac{\partial^{2} v^{\star}}{\partial z^{\star 2}}\right), \\
\frac{\partial w^{\star}}{\partial t^{\star}}+v^{\star} \frac{\partial w^{\star}}{\partial y^{\star}}+w^{\star} \frac{\partial w^{\star}}{\partial z^{\star}}=-\frac{1}{\rho} \frac{\partial p^{\star}}{\partial z^{\star}}+v\left(\frac{\partial^{2} w^{\star}}{\partial y^{\star 2}}+\frac{\partial^{2} w^{\star}}{\partial z^{\star 2}}\right), \\
\frac{\partial T}{\partial t^{\star}}+v^{\star} \frac{\partial T}{\partial y^{\star}}+w^{\star} \frac{\partial T}{\partial z^{\star}}=\frac{K}{\rho C_{p}}\left(\frac{\partial^{2} T}{\partial y^{\star 2}}+\frac{\partial^{2} T}{\partial z^{\star 2}}\right)
\end{gathered}
$$


where $\rho$ is the density, $p^{\star}$ is the fluid pressure, $g$ is the acceleration due to gravity, the coefficient of thermal expansion is $\beta$, the coefficient of heat conduction is $K$, the specific heat at constant pressure is $C_{p}$.

The boundary conditions of the problem are

$$
\begin{gathered}
u^{\star}=0, \quad v^{\star}=-V_{0}\left[1+\epsilon \cos \left(\frac{\pi u_{\infty} z^{\star}}{v}-c t^{\star}\right)\right], \quad w^{\star}=0, \quad T=T_{w} \quad \text { at } y^{\star}=0, \\
u^{\star}=u_{\infty}, \quad v^{\star}=-V_{0}, \quad w^{\star}=0, \quad p^{\star}=p_{\infty}, \quad T=T_{\infty} \quad \text { at } y^{\star}=\infty .
\end{gathered}
$$

Introduce the nondimensional variables

$$
\begin{aligned}
& y=\frac{u_{\infty} y^{\star}}{v}, \quad z=\frac{u_{\infty} z^{\star}}{\infty}, \quad t=c t^{\star}, \quad p=\frac{p^{\star}}{\rho u_{\infty}^{2}}, \\
& u=\frac{u^{\star}}{u_{\infty}}, \quad v=\frac{v^{\star}}{u_{\infty}}, \quad w=\frac{w^{\star}}{u_{\infty}}, \quad \theta=\frac{\left(T-T_{\infty}\right)}{T_{w}-T_{\infty}} .
\end{aligned}
$$

Using (2.8), (2.2)-(2.6) become

$$
\begin{gathered}
\frac{\partial v}{\partial y}+\frac{\partial w}{\partial z}=0 \\
\omega \frac{\partial u}{\partial t}+v \frac{\partial u}{\partial y}+w \frac{\partial u}{\partial z}=\frac{\partial^{2} u}{\partial y^{2}}+\frac{\partial^{2} u}{\partial z^{2}}+G_{r} \theta \\
\omega \frac{\partial v}{\partial t}+v \frac{\partial v}{\partial y}+w \frac{\partial v}{\partial z}=-\frac{\partial p}{\partial y}+\left(\frac{\partial^{2} v}{\partial y^{2}}+\frac{\partial^{2} v}{\partial z^{2}}\right), \\
\omega \frac{\partial w}{\partial t}+v \frac{\partial w}{\partial y}+w \frac{\partial w}{\partial z}=-\frac{\partial p}{\partial z}+\left(\frac{\partial^{2} w}{\partial y^{2}}+\frac{\partial^{2} w}{\partial z^{2}}\right), \\
\omega \frac{\partial \theta}{\partial t}+v \frac{\partial \theta}{\partial y}+w \frac{\partial \theta}{\partial z}=\frac{1}{\operatorname{Pr}}\left(\frac{\partial^{2} \theta}{\partial y^{2}}+\frac{\partial^{2} \theta}{\partial z^{2}}\right)
\end{gathered}
$$

where $\mathrm{Gr}=g \beta\left(T_{w}-T_{\infty}\right) \nu / u_{\infty}^{3}$ is the Grashof number, $\omega=c \nu / u_{\infty}^{2}$ is the frequency parameter, and $\operatorname{Pr}=\rho \nu C_{p} / K$ is the Prandtl number. $T_{\infty}$ is the temperature outside the boundary layer, $p_{\infty}$ is pressure outside the boundary layer.

The boundary conditions (2.7) become

$$
\begin{gathered}
u=0, \quad w=0, \quad v=-S[1+\epsilon \cos (\pi z-t)], \quad \theta=1 \quad \text { at } y=0, \\
u=1, \quad w=0, \quad v=-S, \quad \theta=0 \quad \text { at } y \longrightarrow \infty,
\end{gathered}
$$

where $S=V_{0} / u_{\infty}$ is the suction parameter. 


\section{Flow past a vertical porous plate}

\section{Solution of the problem}

To solve (2.9)-(2.13), we assume the solution of the following form:

$$
\begin{aligned}
& u=u_{0}+\epsilon u_{1}+\epsilon^{2} u_{2}+\cdots, \\
& v=v_{0}+\epsilon v_{1}+\epsilon^{2} v_{2}+\cdots, \\
& w=w_{0}+\epsilon w_{1}+\epsilon^{2} w_{2}+\cdots, \\
& p=p_{0}+\epsilon p_{1}+\epsilon^{2} p_{2}+\cdots, \\
& \theta=\theta_{0}+\epsilon \theta_{1}+\epsilon^{2} \theta_{2}+\cdots,
\end{aligned}
$$

Substituting (3.1) in (2.9)-(2.13), comparing the term free from $\epsilon$ and the coefficient of $\epsilon$ from both sides, and neglecting those of $\epsilon^{2}$, the term free from $\epsilon$ is

$$
\begin{gathered}
v_{0}^{\prime}=0, \\
u_{0}^{\prime \prime}-v_{0} u_{0}^{\prime}+\operatorname{Gr} \theta_{0}=0, \\
\theta_{0}^{\prime \prime}-v_{0} \operatorname{Pr} \theta_{0}^{\prime}=0,
\end{gathered}
$$

where the primes denote differentiation with respect to $y$.

The boundary conditions are

$$
\begin{gathered}
u_{0}=0, \quad v_{0}=-S, \quad \theta_{0}=1 \quad \text { at } y=0, \\
u_{0}=1, \quad v_{0}=-S, \quad \theta_{0}=0 \quad \text { at } y \longrightarrow \infty .
\end{gathered}
$$

The solutions of (3.2)-(3.4) under the boundary conditions (3.5) are

$$
\begin{aligned}
v_{0}(y) & =-S, \quad \theta_{0}(y)=e^{-S \operatorname{Pr} y}, \\
u_{0}(y) & =\left(1-e^{-S y}\right)-\frac{-\mathrm{Gr}}{S^{2} \operatorname{Pr}(\operatorname{Pr}-1.0)}\left(e^{-S \operatorname{Pr} y}-e^{-S y}\right) \quad \text { for } \operatorname{Pr} \neq 1, \\
& =1-e^{-S y}+\frac{\mathrm{Gr}}{S} y e^{-S y} \quad \text { for } \operatorname{Pr}=1.0 .
\end{aligned}
$$

Equating the coefficient of $\epsilon$ from both sides, we get

$$
\begin{aligned}
\frac{\partial v_{1}}{\partial y}+\frac{\partial w_{1}}{\partial z} & =0 \\
\omega \frac{\partial u_{1}}{\partial t}+v_{1} \frac{\partial u_{0}}{\partial y}-S \frac{\partial u_{1}}{\partial y} & =\frac{\partial^{2} u_{1}}{\partial y^{2}}+\frac{\partial^{2} u_{1}}{\partial z^{2}}+\operatorname{Gr} \theta_{1} \\
\omega \frac{\partial v_{1}}{\partial t}-S \frac{\partial v_{1}}{\partial y} & =-\frac{\partial p_{1}}{\partial y}+\left(\frac{\partial^{2} v_{1}}{\partial y^{2}}+\frac{\partial^{2} v_{1}}{\partial z^{2}}\right) \\
\omega \frac{\partial w_{1}}{\partial t}-S \frac{\partial w_{1}}{\partial y} & =-\frac{\partial p_{1}}{\partial z}+\left(\frac{\partial^{2} w_{1}}{\partial y^{2}}+\frac{\partial^{2} w_{1}}{\partial z^{2}}\right), \\
\omega \frac{\partial \theta_{1}}{\partial t}+v_{1} \frac{\partial \theta_{0}}{\partial y}-S \frac{\partial \theta_{1}}{\partial y} & =\frac{1}{\operatorname{Pr}}\left(\frac{\partial^{2} \theta_{1}}{\partial y^{2}}+\frac{\partial^{2} \theta_{1}}{\partial z^{2}}\right) .
\end{aligned}
$$


The boundary conditions become

$$
\begin{aligned}
& u_{1}=0, \quad v_{1}=-S \cos (\pi z-t), \quad w_{1}=0, \quad \theta_{1}=0 \quad \text { at } y=0, \\
& u_{1}=0, \quad v_{1}=0, \quad w_{1}=0, \quad \theta_{1}=0, \quad p_{1}=0 \quad \text { at } y \longrightarrow \infty .
\end{aligned}
$$

These are the linear partial differential equations describing the three-dimensional flow. We assume the velocity components, pressure, and temperature in the following form:

$$
\begin{aligned}
u_{1}(y, z, t) & =u_{11}(y) e^{i(\pi z-t)}, \\
v_{1}(y, z, t) & =v_{11}(y) e^{i(\pi z-t)}, \\
w_{1}(y, z, t) & =\frac{i}{\pi} v_{11}^{\prime}(y) e^{i(\pi z-t)}, \\
p_{1}(y, z, t) & =p_{11}(y) e^{i(\pi z-t)}, \\
\theta_{1}(y, z, t) & =\theta_{11}(y) e^{i(\pi z-t)} .
\end{aligned}
$$

Substituting (3.13) in (3.7)-(3.11), we get the following set of differential equations:

$$
\begin{aligned}
v_{11}^{\prime \prime}+S v_{11}^{\prime}-\left(\pi^{2}-i \omega\right) v_{11} & =p_{11}^{\prime}, \\
v_{11}^{\prime \prime}+S v_{11}^{\prime \prime}-\left(\pi^{2}-i \omega\right) v_{11}^{\prime} & =\pi^{2} p_{11}, \\
\theta_{11}^{\prime \prime}+S \operatorname{Pr} \theta_{11}^{\prime}-\left(\pi^{2}-i \operatorname{Pr} \omega\right) \theta_{11} & =\operatorname{Pr} v_{11} \theta_{0}^{\prime}, \\
u_{11}^{\prime \prime}+S u_{11}^{\prime}-\left(\pi^{2}-i \omega\right) u_{11}^{\prime} & =v_{11} u_{0}^{\prime}-\operatorname{Gr} \theta_{11} .
\end{aligned}
$$

The boundary conditions become

$$
\begin{aligned}
& u_{11}=0, \quad v_{11}=-S, \quad w_{11}=0, \quad \theta_{11}=0 \quad \text { at } y=0, \\
& u_{11}=0, \quad v_{11}=0, \quad w_{11}=0, \quad \theta_{11}=0 \quad \text { at } y \longrightarrow \infty .
\end{aligned}
$$

Solving (3.14)-(3.17), under the boundary conditions (3.18), we get

$$
\begin{aligned}
& v_{1}(y, z)=\frac{S}{\left(\pi-r_{1}\right)}\left[r_{1} e^{-\pi y}-\pi e^{-r_{1} y}\right] e^{i(\pi z-t)}, \\
& w_{1}(y, z)=\frac{i S r_{1}}{\left(\pi-r_{1}\right)}\left[e^{-r_{1} y}-e^{-\pi y}\right] e^{i(\pi z-t)}, \\
& p_{1}(y, z)=\frac{S r_{1}}{\pi\left(\pi-r_{1}\right)}(S \pi-i \omega) e^{-\pi y} e^{i(\pi z-t)}, \\
& \theta_{1}(y, z)=\frac{S^{2} \operatorname{Pr}^{2}}{\left(\pi-r_{1}\right)}\left[C e^{-r_{2} y}-\frac{r_{1}}{\operatorname{Pr}(S \pi+i \omega)} e^{-(\pi+S \operatorname{Pr}) y}\right. \\
& \left.\quad+\frac{\pi}{S r_{1}(1+\operatorname{Pr})+i \omega(\operatorname{Pr}-1.0)} e^{-\left(r_{1}+S \operatorname{Pr}\right) y}\right] e^{i(\pi z-t)},
\end{aligned}
$$




$$
\begin{aligned}
u_{1}(y, z)= & \frac{S^{2}}{\left(\pi-r_{1}\right)}[ \\
& \left.+K_{4} e^{-r_{1} y}+K_{1} e^{-(\pi+S \operatorname{Pr}) y}+K_{5} e^{-\left(r_{1}+S \operatorname{Pr}\right) y}\right] e^{i(\pi z-t)} \quad \text { for } \operatorname{Pr} \neq 1.0 \\
= & \frac{S}{\left(\pi-r_{1}\right)}\left[-\left(C_{2}+C_{3}+C_{4}+C_{5}+\frac{\mathrm{Gr} S B y}{\left(S-2 r_{1}\right)}\right) e^{-r_{1} y}\right. \\
& \left.+\left(C_{2}+C_{4}-\frac{r_{1} \mathrm{Gr} y}{S \pi+i \omega}\right) e^{-(\pi+S) y}+\left(C_{3}+C_{5}+\frac{\pi \mathrm{Gr} y}{2 S r_{1}}\right) e^{-\left(r_{1}+S\right) y}\right] \\
& \times e^{i(\pi z-t)} \quad \text { for } \operatorname{Pr}=1.0,
\end{aligned}
$$

where

$$
\begin{aligned}
& r_{1}=\frac{S+\sqrt{S^{2}+4\left(\pi^{2}-i \omega\right)}}{2}, \quad r_{2}=\frac{S \operatorname{Pr}+\sqrt{S^{2} \operatorname{Pr}^{2}+4\left(\pi^{2}-i \omega \operatorname{Pr}\right)}}{2}, \\
& C=\frac{r_{1}}{\operatorname{Pr}(S \pi+i \omega)}-\frac{\pi}{S r_{1}(\operatorname{Pr}+1)+i \omega(\operatorname{Pr}-1)} \\
& K_{1}=\frac{r_{1}\left(1+C_{1}\right)}{(S \pi+i \omega)}, \quad K_{2}=\frac{-\mathrm{GrPr}^{2} C}{r_{2}^{2}-S r_{2}-\pi^{2}+i \omega}, \quad K_{3}=\frac{-\pi\left(1+C_{1}\right)}{2 S r_{1}}, \\
& K_{4}=\left\{\frac{G r \operatorname{Pr}^{2} r_{1}}{\operatorname{Pr}(S \pi+i \omega)}-r_{1} C_{1} \operatorname{Pr}\right\} /\left\{S^{2} \operatorname{Pr}(\operatorname{Pr}-1)+S \pi(2 \operatorname{Pr}-1.0)+i \omega\right\}, \\
& K_{5}=\left\{\pi C_{1} \operatorname{Pr}-\frac{\pi \mathrm{GrPr}^{2}}{S r_{1}(\operatorname{Pr}+1)+i \omega(\operatorname{Pr}-1)}\right\} /\left\{S \operatorname{Pr}\left(2 r_{1}+S \operatorname{Pr}-S\right)\right\}, \\
& C_{1}=\frac{-\mathrm{Gr}}{S^{2} \operatorname{Pr}(\operatorname{Pr}-1.0)}, \quad C_{2}=\left\{r_{1}\left(S+\frac{\mathrm{Gr}}{S}\right)+\frac{\mathrm{Gr} S r_{1}}{S \pi+i \omega}\right\} \frac{1}{S \pi+i \omega}, \\
& C_{3}=-\left\{\pi\left(S+\frac{\mathrm{Gr}}{S}\right)+\frac{\mathrm{Gr} \pi}{2 r_{1}}\right\} \frac{1}{2 S r_{1}}, \quad C_{4}=\frac{-\mathrm{Gr} r_{1}(2 \pi+S)}{(S \pi+i \omega)^{2}}, \quad C_{5}=\frac{\pi \mathrm{Gr}\left(S+2 r_{1}\right)}{4 S^{2} r_{1}^{2}} \text {. }
\end{aligned}
$$

\section{Result and discussion}

We have presented the nondimensional main flow velocity $u$ and cross-velocity $w$ against $y$ for different values of $\operatorname{Pr}, S$, and $\omega$ and for $\mathrm{Gr}=5.0, z=0.2, t=0.2$. The main flow velocity profiles are shown graphically in Figures 4.1 and 4.2. We observed that the main flow decreases with increase in Prandtl number while it increases with increase in suction parameter. Also we obtained $u$ for different values of $\omega$ which is given in Table 4.1. From the table, it is seen that $u$ increases with increase in $\omega$, but the effect is negligible. From Figure 4.3, it is seen that the cross-flow decreases near the plate and increases away from the plate with increase in suction parameter. Figure 4.4 shows that the magnitude of the cross-flow increases near the plate and decreases away from the plate with increase in frequency parameter $\omega$.

The main velocity $u$ for different values of $\omega$ is shown in the table. 


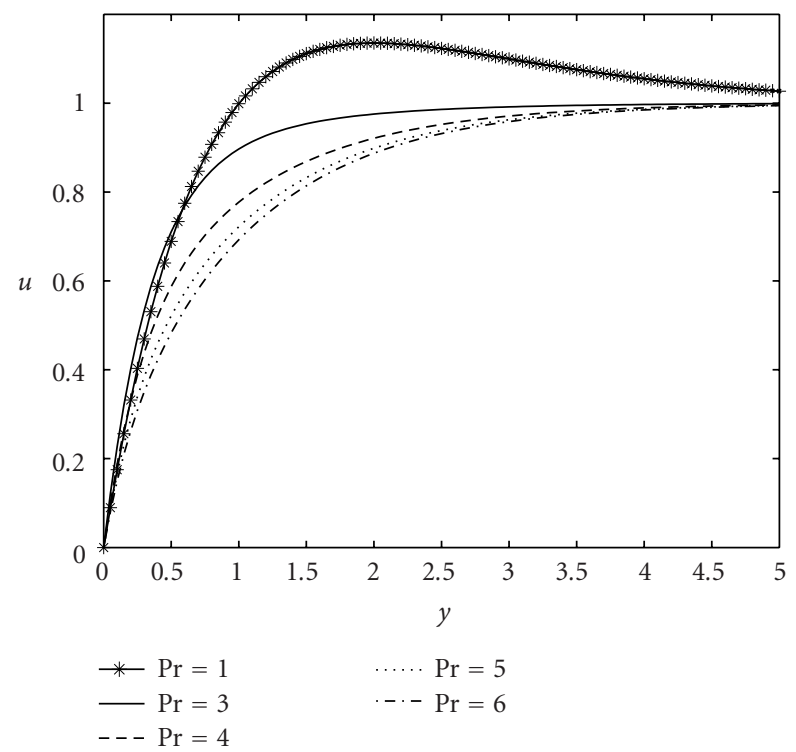

Figure 4.1. Main flow velocity $u$ for $\mathrm{Gr}=5.0, S=1.0, \omega=10, z=0.2, t=0.2, \epsilon=0.2$.

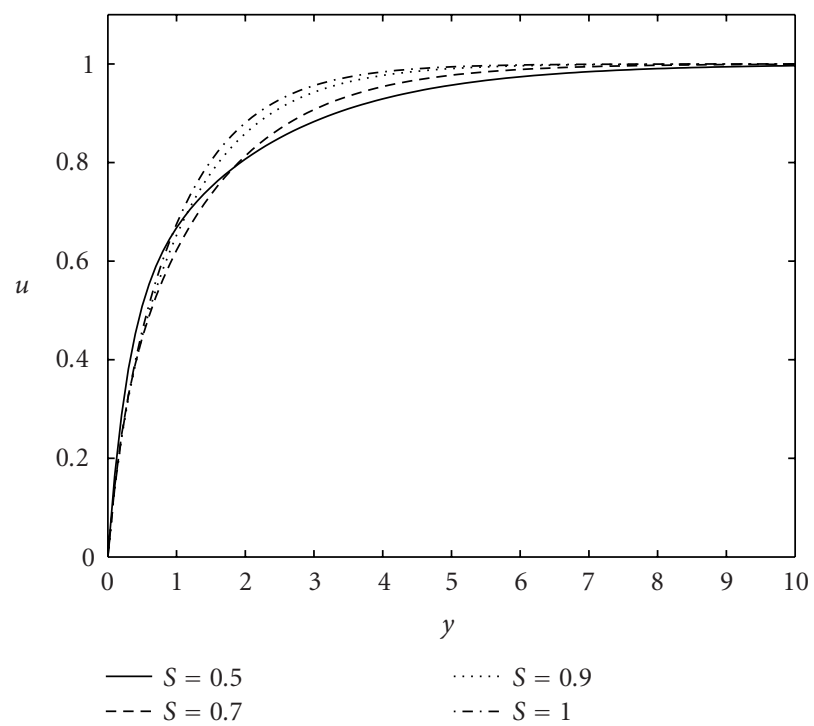

Figure 4.2. Main flow velocity $u$ for $\mathrm{Gr}=5.0, \operatorname{Pr}=7.0, \omega=10, z=0.2, t=0.2, \epsilon=0.2$.

The important characteristic of the problem is shear stress. The shear stress due to main flow direction at the plate $y=0$ is

$$
\tau_{x}=\left(\frac{\partial u}{\partial y}\right)_{y=0}=u_{0}^{\prime}(0)+\epsilon u_{1}^{\prime}(0)=u_{0}^{\prime}(0)+\epsilon u_{11}^{\prime}(0) e^{i(\pi z-t)} .
$$


3366 Flow past a vertical porous plate

Table 4.1. Main velocity $u$ for $\mathrm{Gr}=5.0, \operatorname{Pr}=7.0, S=1.0$, and $z=0.2$.

\begin{tabular}{c|ccccc}
\hline \multirow{2}{*}{$y$} & \multicolumn{5}{|c}{$u$} \\
\cline { 2 - 6 } & $\omega=5$ & $\omega=8$ & $\omega=10$ & $\omega=15$ & $\omega=20$ \\
\hline 0.00 & 0.00000000 & 0.00000000 & 0.00000000 & 0.00000000 & 0.00000000 \\
1.00 & 0.67414890 & 0.67491380 & 0.67526410 & 0.67564240 & 0.67575110 \\
2.00 & 0.88072730 & 0.88076850 & 0.88077700 & 0.88077790 & 0.880777640 \\
3.00 & 0.95613920 & 0.95614020 & 0.95614010 & 0.95614000 & 0.95614000 \\
4.00 & 0.98386490 & 0.98386490 & 0.98386490 & 0.98386490 & 0.98386490 \\
5.00 & 0.99406430 & 0.99406430 & 0.99406430 & 0.99406430 & 0.99405430 \\
6.00 & 0.99781640 & 0.99781640 & 0.99781640 & 0.99781640 & 0.99781640 \\
7.00 & 0.99919670 & 0.99919670 & 0.99919670 & 0.99919670 & 0.99919670 \\
8.00 & 0.99970450 & 0.99970450 & 0.99970450 & 0.99970450 & 0.99970450 \\
9.00 & 0.99989130 & 0.99989130 & 0.99989130 & 0.99989130 & 0.99989130 \\
10.00 & 0.99996010 & 0.99996010 & 0.99996010 & 0.99996010 & 0.99996010 \\
\hline
\end{tabular}

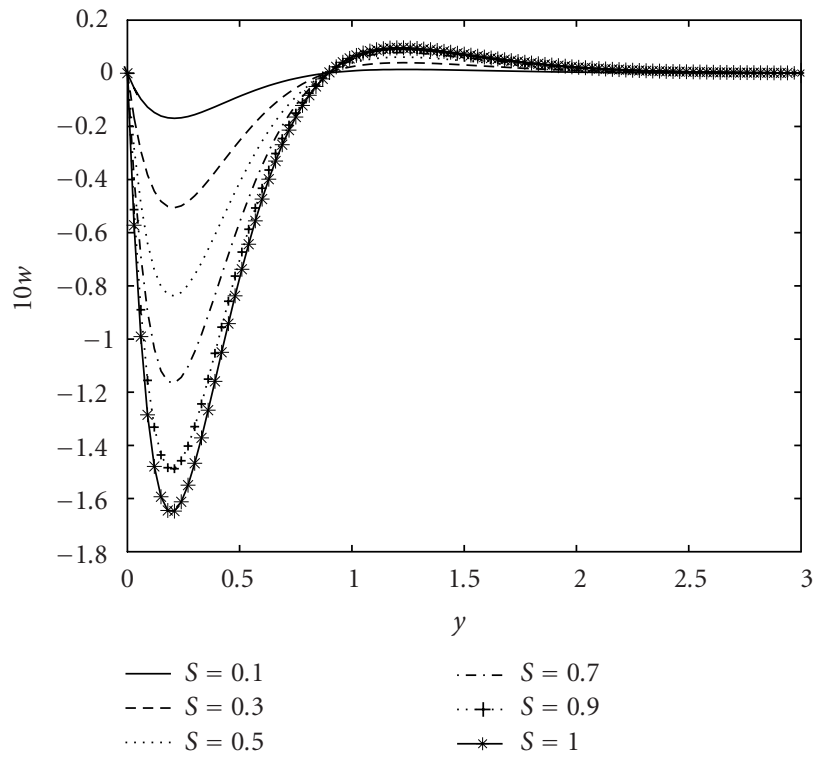

Figure 4.3. Cross-velocity $10 \times w$ for $\mathrm{Gr}=5.0, \omega=10, z=0.2, t=0.2, \epsilon=0.2$.

We express the shear stress component in terms of magnitude and tangent of phase shift:

$$
\begin{array}{ll}
\tau_{x}=u_{0}^{\prime}(0)+\epsilon\left|R_{1}\right| \cos \left(\pi z-t+\phi_{1}\right) & \text { for } \operatorname{Pr} \neq 1.0, \\
\tau_{x}=u_{0}^{\prime}(0)+\epsilon\left|R_{1}^{\prime}\right| \cos \left(\pi z-t+\phi_{1}^{\prime}\right) & \text { for } \operatorname{Pr}=1.0,
\end{array}
$$




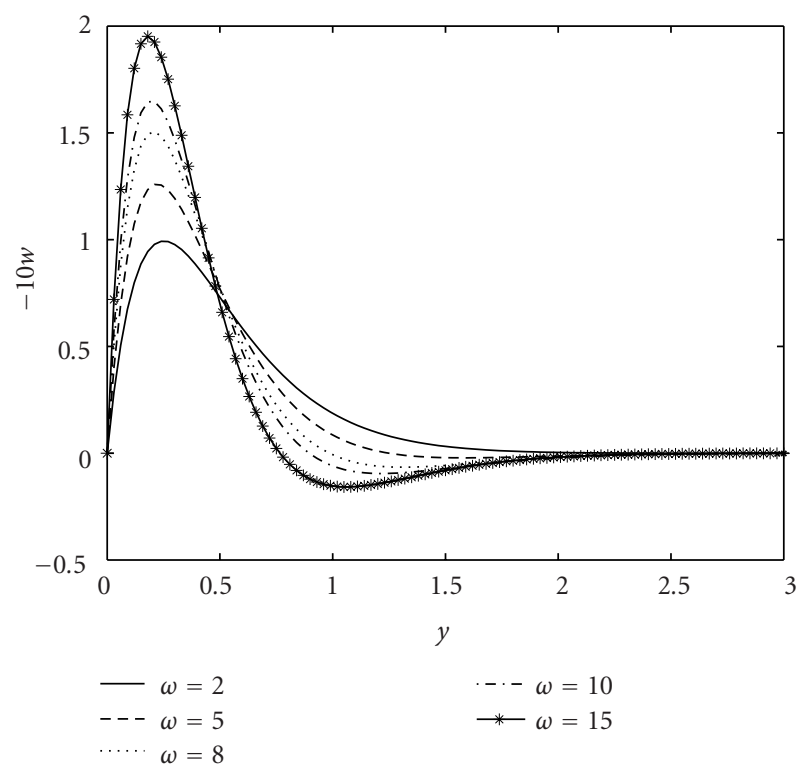

Figure 4.4. Cross-velocity $-10 \times w$ for $\mathrm{Gr}=5.0, S=1.0, z=0.2, t=0.2, \epsilon=0.2$.

where

$$
R_{1}=\sqrt{u_{1 r}^{2}+u_{1 i}^{2}}, \quad \tan \phi_{1}=\frac{u_{1 i}}{u_{1 r}}, \quad R_{2}=\sqrt{u_{11 r}^{2}+u_{11 i}^{2}}, \quad \tan \phi_{2}=\frac{u_{11 i}}{u_{11 r}} .
$$

The magnitude and the tangent of phase shift of the shear stress due to main flow are shown graphically in Figures 4.5, 4.6, 4.7, and 4.8 against $\omega$ for different values of Prandtl number and Grashoff number. It is seen that the magnitude and the tangent of phase shift of the shear stress decrease with increase in either Prandtl number Pr, Grashof number Gr, or frequency parameter $\omega$.

The temperature $\theta$ for different values of $\omega$ is shown in the table.

The temperature distribution has been obtained and plotted for different values of $\mathrm{Pr}$ and $S$ in Figures 4.9 and 4.10 for $\omega=10.0, \mathrm{Gr}=5.0, t=0.2$. It is found that the temperature $\theta$ decreases with increase in either Prandtl number or suction parameter. Also we have obtained the temperature distribution for different values of frequency parameter which is given in Table 4.2. From the table, it is found that the temperature decreases with increase in frequency parameter.

Also, we calculate the rate of heat transfer. The rate of heat transfer at the plate $y=0$ is given by

$$
\begin{aligned}
\left(\frac{\partial \theta_{11}}{\partial y}\right)_{y=0} & =\theta_{0}^{\prime}(0)+\epsilon \theta_{1}^{\prime}(0) \\
& =\theta_{0}^{\prime}(0)+\epsilon \theta_{11}^{\prime}(0) e^{i(\pi z-t)} \\
& =\theta_{0}^{\prime}(0)+\epsilon\left|R_{3}\right| \cos \left(\pi z-t+\phi_{3}\right) .
\end{aligned}
$$




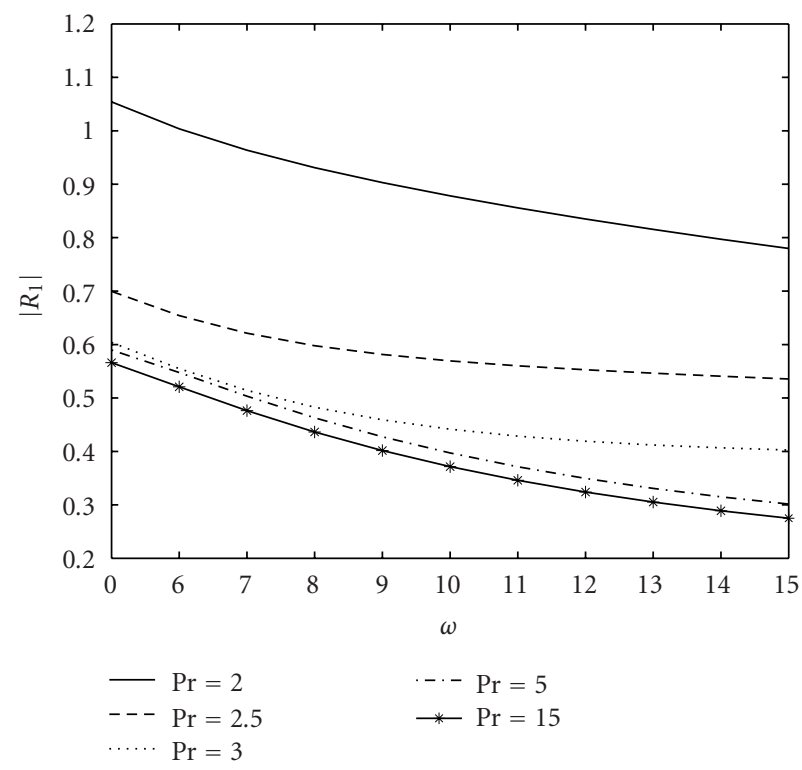

Figure 4.5. Amplitude of the shear stress due to main flow for $\mathrm{Gr}=5.0, S=1.0, z=0.2$.



Figure 4.6. Tangent of phase shift of shear stress due to main flow for $\mathrm{Gr}=5.0, S=1.0, z=0.0$.

We draw the graph of amplitude and tangent of phase shift of the rate of heat transfer against frequency parameter $\omega$ for different values of Prandtl number Pr in Figures 4.11 


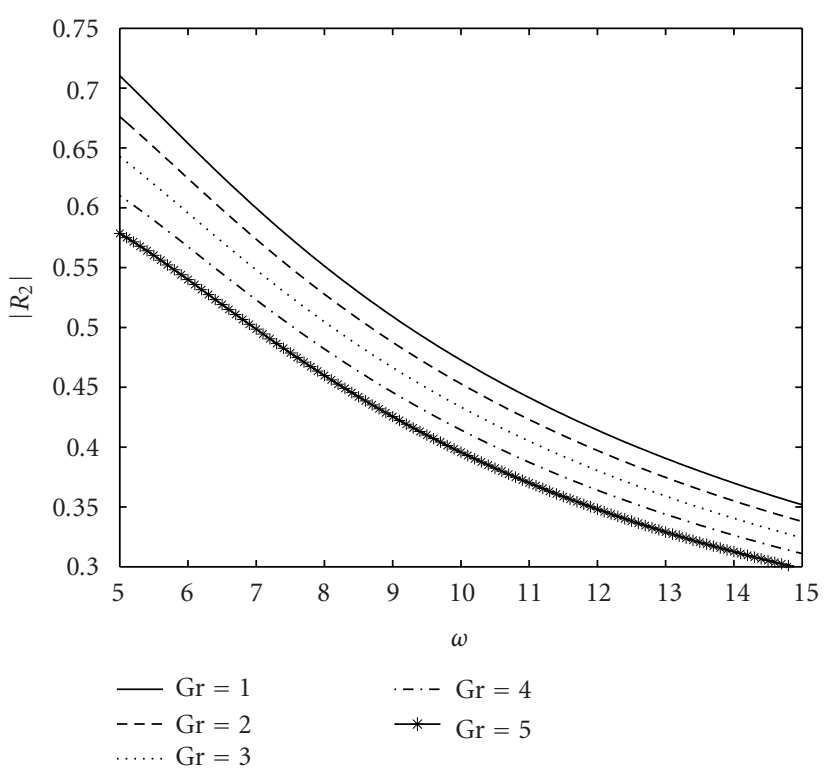

Figure 4.7. Amplitude of the shear stress due to main flow for $S=1.0$.

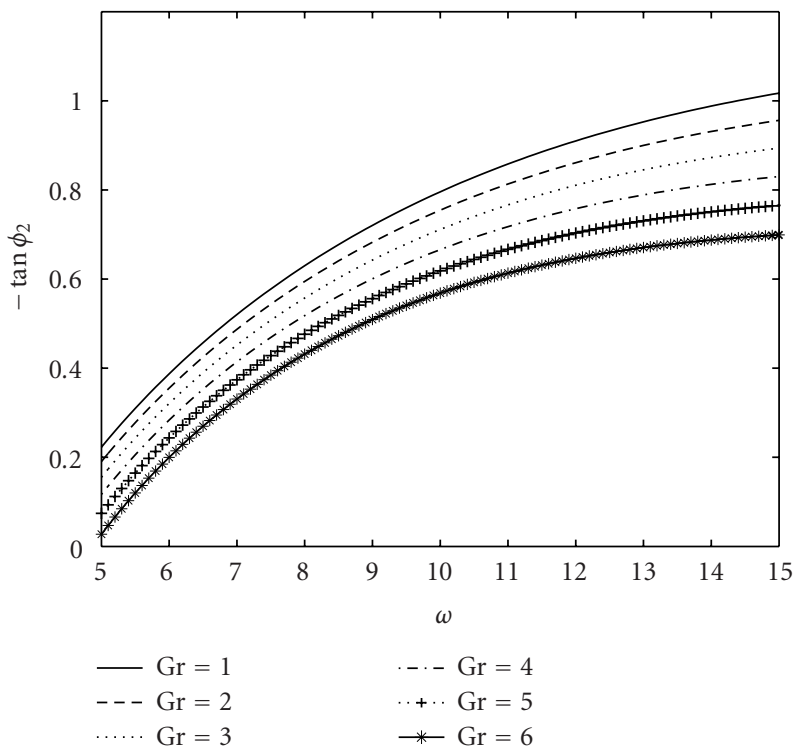

Figure 4.8. Tangent of phase shift of the shear stress due to main flow for $S=1.0$.

and 4.12 and for $\mathrm{Gr}=5.0, S=1.0$. From Figure 4.11, we see that the amplitude increases with increase in Prandtl number but decreases with increase in frequency parameter $\omega$. 


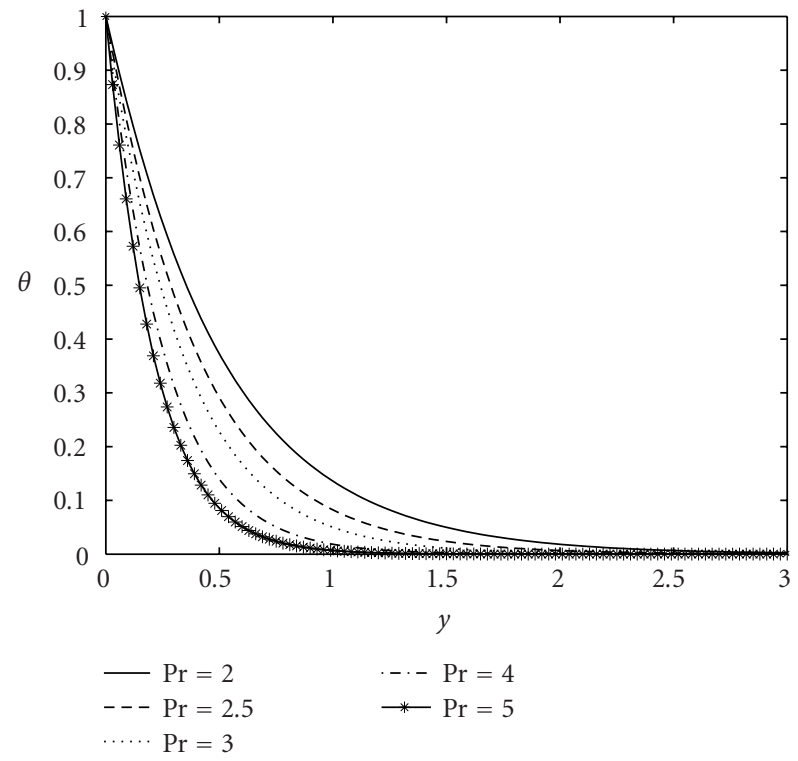

Figure 4.9. Temperature profile for $\mathrm{Gr}=5.0, S=1.0, \omega=10.0, z=0.2, t=0.2, \epsilon=0.2$.

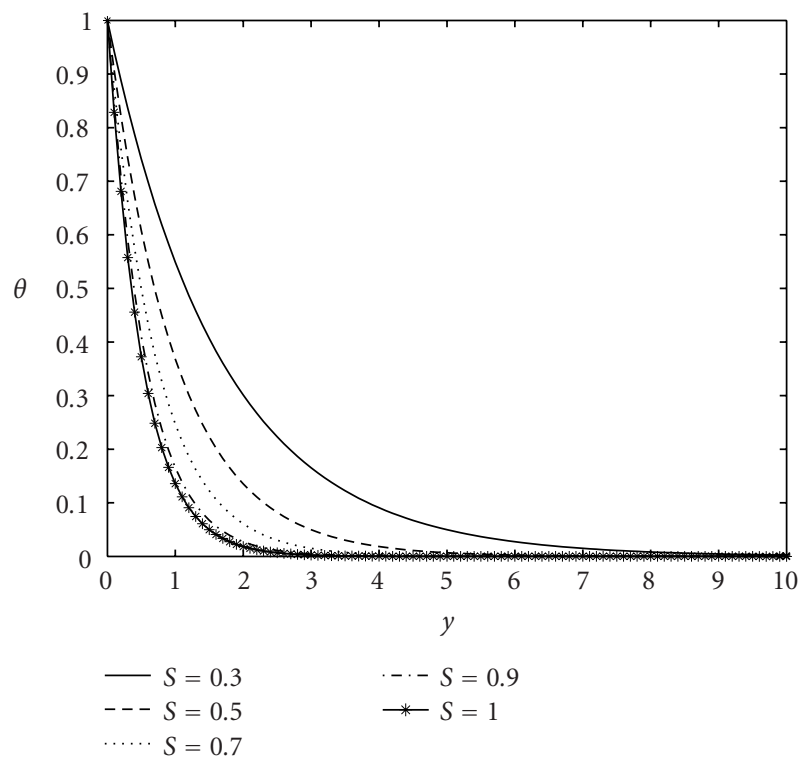

Figure 4.10. Temperature profile for $\mathrm{Gr}=5.0, \operatorname{Pr}=2.0, \omega=10.0, z=0.2, t=0.2, \epsilon=0.2$.

Figure 4.12 shows that the magnitude of tangent of phase shift decreases with increase in Prandtl number Pr but increases with increase in $\omega$. It is seen that there is always a phase lag. 
Table 4.2. Temperature distribution for $\mathrm{Gr}=5.0, S=1.0, \operatorname{Pr}=2.0$.

\begin{tabular}{l|ccccc}
\hline \multirow{2}{*}{$y$} & \multicolumn{5}{|c}{$\theta$} \\
\cline { 2 - 6 } & $\omega=5$ & $\omega=8$ & $\omega=10$ & $\omega=15$ & $\omega=20$ \\
\hline 0.00 & 1.00000000 & 1.00000000 & 1.00000000 & 1.00000000 & 1.00000000 \\
0.40 & 0.46545780 & 0.45846200 & 0.45615440 & 0.45372500 & 0.45261860 \\
0.80 & 0.20575920 & 0.20351230 & 0.20298460 & 0.20245720 & 0.20221060 \\
1.20 & 0.09137923 & 0.09091454 & 0.09083322 & 0.09075849 & 0.09073284 \\
1.60 & 0.04085174 & 0.04077750 & 0.04077017 & 0.04076410 & 0.04076333 \\
2.00 & 0.01832459 & 0.01831620 & 0.01831609 & 0.01831586 & 0.01831588 \\
2.40 & 0.00823015 & 0.00822975 & 0.00822981 & 0.00822979 & 0.00822978 \\
2.80 & 0.00369778 & 0.00369788 & 0.003697888 & 0.00369787 & 0.00369787 \\
3.20 & 0.00166153 & 0.00166156 & 0.00166156 & 0.00166156 & 0.00166156 \\
3.60 & 0.00074658 & 0.00074659 & 0.00074659 & 0.0074659 & 0.00074659 \\
4.00 & 0.00033546 & 0.00033546 & 0.00033546 & 0.00033546 & 0.00033546 \\
\hline
\end{tabular}

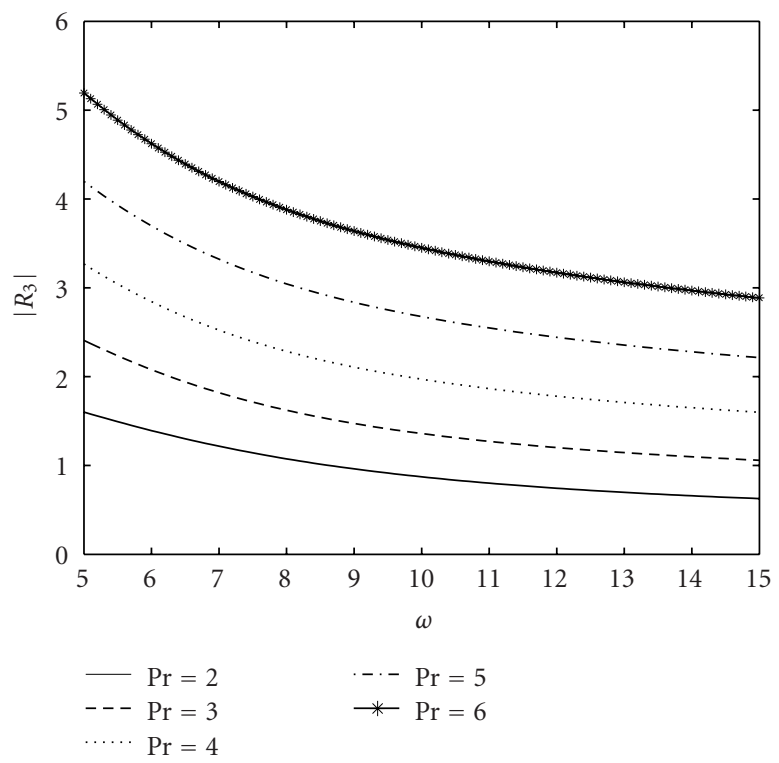

Figure 4.11. Amplitude of the rate of heat transfer for $\mathrm{Gr}=5.0, S=1.0$.

\section{Acknowledgment}

M. Guria is highly thankful to the University Grants Commission (UGC) for granting the fellowship. 




Figure 4.12. Tangent of phase shift of the rate of heat transfer for $\mathrm{Gr}=5.0, S=1.0$.

\section{References}

[1] K. D. Singh, Hydromagnetic effects on the three-dimensional flow past a porous plate, Z. Angew. Math. Phys. 41 (1990), no. 3, 441-446.

[2] , Three-dimensional viscous flow and heat transfer along a porous plate, Z. Angew. Math. Mech. 73 (1993), no. 1, 58-61.

[3] P. Singh, V. P. Sharma, and U. N. Misra, Three dimensional fluctuating flow and heat transfer along a plate with suction, Int. J. Heat Mass Transfer 21 (1978), 1117-1123.

[4] Three dimensional free convection flow and heat transfer along a porous vertical plate, Appl. Sci. Res. 34 (1978), no. 1, 105-115.

M. Guria: Department of Applied Mathematics with Oceanology and Computer Programming, Vidyasagar University, Midnapore 721 102, West Bengal, India

E-mail address: mrinmoy9832@yahoo.com

R. N. Jana: Department of Applied Mathematics with Oceanology and Computer Programming, Vidyasagar University, Midnapore 721 102, West Bengal, India

E-mail address: jana261171@yahoo.co.in 


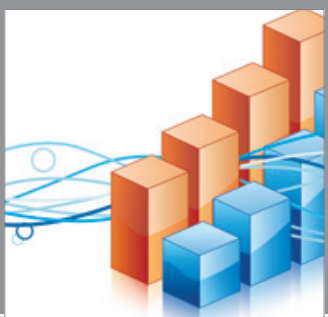

Advances in

Operations Research

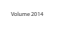

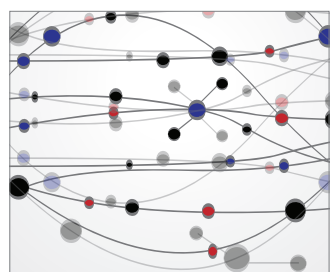

\section{The Scientific} World Journal


International Journal of

Mathematics and

Mathematical

Sciences
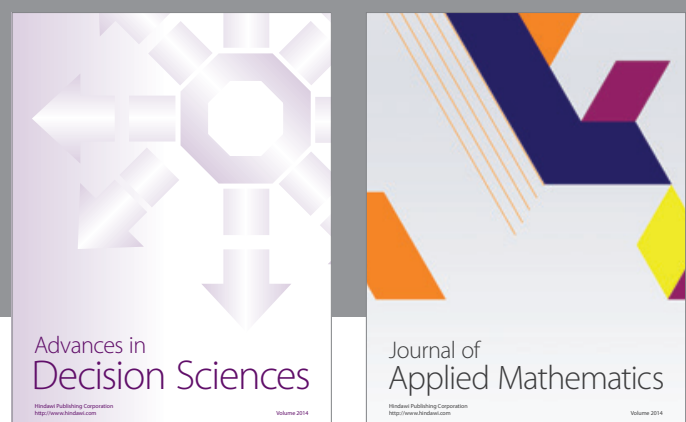

Journal of

Applied Mathematics


Submit your manuscripts at http://www.hindawi.com
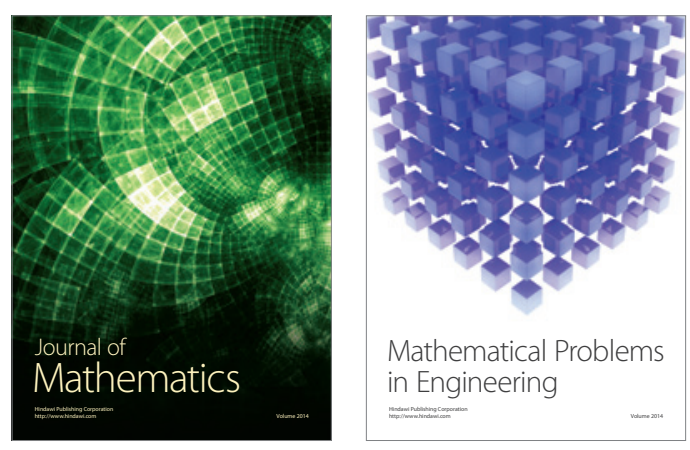

Mathematical Problems in Engineering
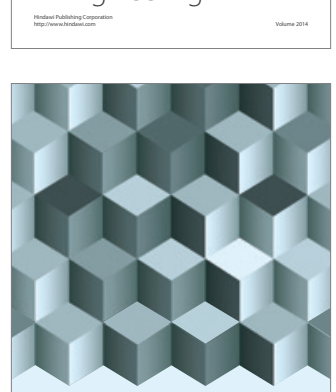

Journal of

Function Spaces
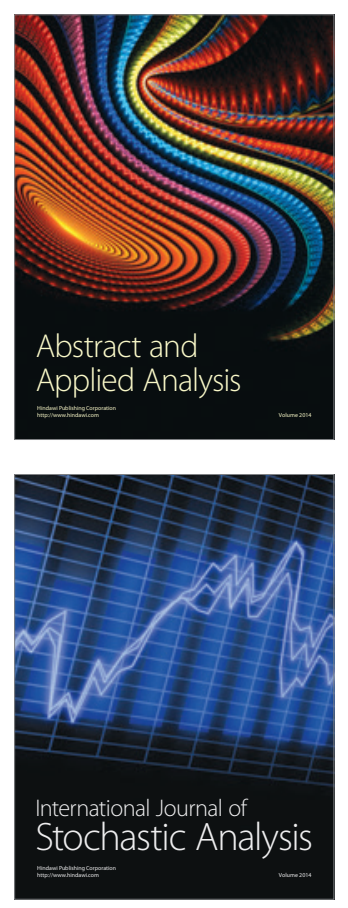



ournal of

Probability and Statistics

Promensencen
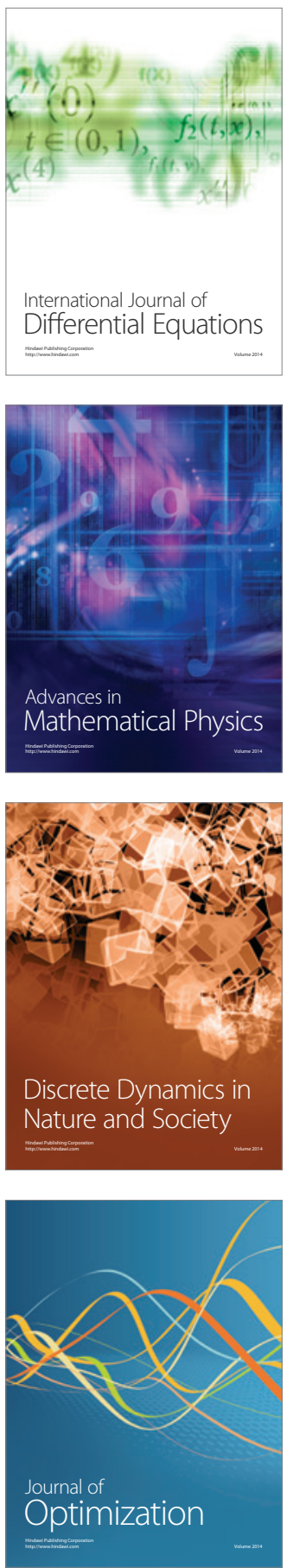